\title{
Desafíos de la política pública colombiana frente a la transferencia de resultados de investigación universitaria ${ }^{1}$
}

\author{
Challenges Raised by the Colombian Public policy Regarding the Transfer of \\ University Research Results
}

\author{
Leonardo Andrés Briceño Marín ${ }^{2}$ \\ Maria Eugenia Morales Rubiano ${ }^{3}$
}

Fecha de recepción: 23 de noviembre de 2014

Fecha de aprobación: 4 de abril de 2015

\section{RESUMEN}

El objetivo de este artículo es identificar los desafíos que tiene la política pública colombiana frente a la transferencia de resultados de investigación universitaria al entorno. Se revisaron políticas relacionadas, extraídas de artículos indexados y páginas web institucionales de países representativos en materia de ciencia y tecnología de Europa, Asía, Norteamérica y Latinoamérica. Se encontró que en países industrializados hay un fuerte vínculo entre la universidad y el sector productivo; mientras que en Colombia, aun cuando el gobierno ha generado un marco de políticas para fomentar la ciencia, tecnología e innovación, hace falta destinar más recursos en estas actividades, mejorar la distribución de los mismos y fortalecer los mecanismos de coordinación entre actores del Sistema Nacional de Innovación (SNI).

1 Artículo producto del proyecto de investigación No. INV ECO-1782: "Fortalecimiento de capacidades para la innovación de las micro y pequeńas empresas asociadas a Fenalco en Bogotá y Pereira”. Financiado por la Vicerrectoría de Investigaciones de la Universidad Militar Nueva Granada.

2 Estudiante de Maestría en Gestión de Organizaciones, Facultad Ciencias Económicas, Universidad Militar Nueva Granada, Colombia. Correo electrónico: tmp.leonardo.briceno@unimilitar.edu.co. Vigencia 2015

3 Profesora, Facultad de Ciencias Económicas, Universidad Militar Nueva Granada, Colombia, Magíster en Administración. Correo electrónico: maria.morales@unimilitar.edu.co 
Palabras clave: políticas públicas de ciencia, tecnología e innovación, transferencia de conocimiento universitario.

\begin{abstract}
The aim of this paper is to identify the challenges that Colombian public policy faces regarding the transfer of research results from universities to their surroundings. Related policies, extracted from indexed articles and institutional web pages of representative countries in science, technology and innovation in Europe, Asia, North America and Latin America, were reviewed. It was found that in industrialized countries there is a strong link between university and the productive sector; while in Colombia, even though the government has created a policy framework to promote science, technology and innovation, it is needed to allocate more resources to these activities, improve their distribution thereof, and strengthen coordination mechanisms between actors of the National Innovation System (SIN, for its abbreviation in Spanish).
\end{abstract}

Keywords: Public policies of science, technology and innovation, university knowledge transfer.

\title{
INTRODUCCIÓN
}

Las políticas públicas son una tarea colectiva de iniciativa social y gubernamental como respuesta a una problemática (Aguilar, 1996, citado en Herrera \& Morales, 2014). En cuanto a ciencia (C), tecnología (T), investigación (I), desarrollo (D) e innovación (i), Colombia tiene dificultades por no tener una $\mathrm{C}$ y $\mathrm{T}$ competitiva a nivel mundial y destinar bajos niveles de inversión en $\mathrm{I}+\mathrm{D}+\mathrm{i}$. Las políticas de $\mathrm{C}$ y $\mathrm{T}$ se establecen para mejorar la capacidad tecnológica y productiva del país, también para fomentar la transferencia de conocimiento. En este sentido, es importante realizar el ejercicio de identificar los desafíos que tiene la política pública colombiana frente a la transferencia de resultados de investigación universitaria, tomando como ejemplo el éxito en otros paises. 
Leonardo Andrés Briceño Marín, María Eugenia Morales Rubiano Desafíos de la política pública colombiana frente a la transferencia de resultados de investigación universitaria

La evolución de la economía está centrada en las funciones de la infraestructura del conocimiento de sistemas industriales competitivos, los cuales necesitan que el gobierno adopte adecuadas políticas de I+D (Etzkowitz \& Leydesdorff, 1995).

La sociedad del conocimiento subsiste del aprendizaje del desarrollo científico y tecnológico (Corbí, 1992), en donde el conocimiento es un recurso económico clave que transforma a la sociedad (Drucker, 1993). Para adentrase a la sociedad del conocimiento, es necesario establecer estrategias para ser competitivo en una economía globalizada y el gobierno es el actor fundamental para favorecer el desarrollo de la $\mathrm{C}+\mathrm{T}+\mathrm{I}+\mathrm{D}+\mathrm{i}$. Sin la participación de éste es muy complicado que puedan surgir resultados óptimos de la transferencia de investigación.

El objetivo de este artículo es identificar los desafíos que tiene la política pública colombiana frente a la transferencia de resultados de investigación universitaria al sector productivo, el gobierno y la sociedad. Con lo anterior, se busca contribuir a mostrar cómo se han configurado las políticas públicas en Colombia, relacionadas con la promoción de la $\mathrm{C}+\mathrm{T}+\mathrm{I}+\mathrm{D}+\mathrm{i}$ y la transferencia de investigación desde la universidad a su entorno, y cuáles son los retos más importantes que todavía tiene el país en este campo.

\section{TRANSFERENCIA DE CONOCIMIENTO Y TRANSFERENCIA DE TECNOLOGÍA}

El proceso en donde el emisor transfiere su conocimiento al receptor se denomina transferencia de conocimiento (Dawson, 2000). Puede ser directo, a través de interacciones entre los actores o indirecto, en donde el conocimiento especializado se transforma en información comprensible, normalmente en documentos, planos o medios digitales (Dawson, 2000). Esta transferencia es una herramienta estratégica que brinda ventajas competitivas, mejorando los procesos de la organización receptora y a su vez la emisora (Cubides \& Vélez, 2010). 
El concepto de transferencia ha estado ligado a la administración de tecnología, pero se ha aceptado globalmente como transferencia de conocimiento, en donde se le da más valor al conocimiento de las personas en una organización. A su vez, la transferencia de tecnología se diferencia entre dos grupos, uno es el institucional, el cual es referente a la universidad y el individual, referente al investigador (Aceytuno \& Sánchez-López, 2014). Conceptualmente, la transferencia de tecnología es la entrega de información tecnológica o tecnología (Chile, Ministerio de Economía, Fomento y Turismo, 2014).

La investigación científica se relaciona con la tecnológica, ambas dependen una de la otra. Las dos generan conocimiento. La sociedad debe transformarse hacia la producción de conocimiento y la riqueza, la cual ya no se estipula en poseer bienes sino conocimiento (Corbí, 1992).

La transferencia de conocimiento entre organizaciones optimiza recursos. La que recibe, aprovecha la experiencia de la generadora (Mendoza, 1996, citado en Cubides \& Vélez, 2010). Así, la integración estratégica en los proyectos de innovación tecnológica de la relación universidad-empresa facilita la gestión organizada del conocimiento (Llanos \& Dager, 2003).

\section{Modelos lineales de transferencia de tecnología y conocimiento}

Se caracterizan por manejarse como una línea y tener secuencias. El modelo de empuje es el que empieza desde la fuente de la innovación pasando por la investigación, el desarrollo, la fabricación y la comercialización del descubrimiento científico. El modelo de tirón de la demanda se basa en las necesidades del mercado, de allí se desprende el desarrollo, la producción y termina por la comercialización del desarrollo científico (Rothwell, 1994). La transferencia de resultados de investigación desde las universidades a su entorno estuvo inicialmente centrada en el modelo de empuje. Pero los requerimientos de un contexto globalizado y altamente competitivo, han llevado a que actualmente estos procesos estén sujetos al jalón de la demanda y a modelos más dinámicos e interactivos. 
Leonardo Andrés Briceño Marín, María Eugenia Morales Rubiano Desafíos de la política pública colombiana frente a la transferencia de resultados de investigación universitaria

\section{Modelos interactivos de transferencia entre universidad y su entorno}

Las organizaciones son conformadas por personas que trabajan por un fin en común; un fin de la organización es hacer productivo los conocimientos (Drucker, 1993). La integración entre los agentes del sistema económico facilita la transferencia de conocimiento desde las universidades hacia el sector productivo (Muñoz, 2010) y contribuye con el desarrollo económico (Melero, Angulo, \& Martín, 2011). Actualmente, existen diferentes planteamientos que buscan mostrar y explicar las relaciones que ocurren entre actores de un SNI en un proceso de transferencia.

Los sistemas de innovación son redes de instituciones públicas y privadas, las cuales interactúan, empiezan, importan, modifican y transfieren desarrollos tecnológicos (Freeman, 1987, citado en Muñoz, 2005). Estos se dividen en sistemas nacionales y regionales. Los primeros generalmente provienen de los regionales y se enmarcan en la globalización. Los segundos se establecen como un fenómeno regional y sistémico (Cooke, 1998 citado en Pineda, Morales, \& Ortiz, 2011).

Algunos autores han propuesto modelos que buscan explicar la relación de actores dentro de un SNI. Uno de estos planteamientos es el Triángulo de Sábato, el cual muestra la relación entre gobierno, la infraestructura científico-tecnológica y la estructura productiva, en donde se asegura la capacidad racional de la sociedad para innovar. El vértice gobierno formula e implementa políticas concernientes a la $\mathrm{C}$ y la T. El vértice de la infraestructura científico-tecnológica es la capacidad creadora de la investigación científica. La estructura productiva está compuesta por las empresas del sector público y del sector privado (Sabato \& Botana, 1968).

El gobierno se relaciona con la infraestructura científico-tecnológica a través de la asignación de recursos y del impulso de la innovación científica. A su vez, la infraestructura científico-tecnológica satisface la demanda tecnológica y la estructura productiva también provee de recursos a la infraestructura científico-tecnológica (Sabato \& Botana, 1968). Este modelo se muestra como una relación de forma triangular en donde se sitúan los actores en los vértices que a través de relaciones promueven la $\mathrm{I}+\mathrm{D}+\mathrm{i}$. 
De la misma manera, la universidad y la industria no son esferas separadas: tienen una relación con el gobierno para propulsar la innovación. Esta relación fue denominada la Triple Hélice, la cual es un componente clave de la estrategia de los países porque los tres actores trabajan conjuntamente facilitando los procesos de desarrollo tecnológico y científico a través de la colaboración y la integración, logrando que los resultados sean más dinámicos (Etzkowitz \& Leydesdorff, 1995). Lo anterior se puede promover a través de la formulación e implementación de correctas políticas públicas de $\mathrm{C}$ y $\mathrm{T}$. Además, para poder impulsar la $\mathrm{I}+\mathrm{D}+\mathrm{i}$, es necesario que hayan actores quienes financien y demanden las invenciones.

La relación que tiene la universidad para conectarse con la sociedad a través de la transferencia de actividades de investigación es llamada la tercera misión. Ésta hace que la universidad salga de su papel tradicional de formar a los estudiantes y de desarrollar procesos de investigación (Laredo, 2007; Aceytuno \& Cáceres, 2009; Clark, 2001). Por medio de la tercera misión, la universidad aumenta la relación con el sector productivo para contribuir con el desarrollo de la tecnología e incremento de la economía por medio de actividades de transferencia de conocimiento (Calderón y García, 2013).

De esta forma, la transferencia de conocimiento se convierte en la tercera misión universitaria, poniéndola como impulsadora de la innovación y dándole el papel de generar, aplicar y explotar el conocimiento desde la universidad hacia el entorno (Melero et al., 2011). La promulgación de la tercera misión se da en las páginas web de las universidades, a través de indicadores y cifras como: egresados, patentes concedidas, publicaciones, ingresos por contratos y número de empresas de spin off (Laredo, 2007).

\section{Medios o canales de transferencia de resultados de investigación}

Según la motivación para participar en los vínculos y hacia dónde fluye el conocimiento, los canales de interacción se clasifican en cuatro tipos (ver tabla 1): (a) el canal tradicional, en donde el conocimiento se dirige principalmente desde los centros de investigación hacia el sector productivo por los roles convencionales, 
como ejemplo la enseñanza e investigación; (b) el canal de servicios, el cual motiva los servicios científicos a cambio de dinero, por ejemplo las consultorías; (c) el canal comercial, el cual busca comercializar los resultados de investigación de los centros de investigación; y (d) el canal bidireccional pretende crear conocimiento a través del desarrollo de contratos de $\mathrm{I}+\mathrm{D}$, proyectos y redes a largo plazo, por ejemplo. En los tres primeros canales fluye principalmente el conocimiento desde los centros de investigación hacia el sector productivo y el último fluye en ambos sentidos (Dutrénit, Fuentes, \& Torres, 2010).

Tabla 1. Canales de interacción entre centros de investigación y sector productivo

\section{Canales}

Formas

Creación de redes con el sector productivo

Bidireccional

$$
\text { Proyectos conjuntos de I + D }
$$

Contratos de investigación

\begin{tabular}{ll} 
& Patentes \\
\hline Comercial & Licencias de Tecnología \\
\hline Incubadoras \\
\hline Spin off \\
\hline Movilidad de personal
\end{tabular}

Servicios

Consultoría y asistencia técnica

Intercambio informal de información

Conferencias y exposiciones

Tradicional

Publicaciones

Recién graduados empleados en la industria

Fuente: Dutrénit, G., De Fuentes, C., \& Torres, A. (2010). Channels of interaction between public research organisations and industry and their benefits: evidence from Mexico. Science \& Public Policy (SPP), 37(7), 513526. doi:10.3152/030234210X512025 
Lo más básico que hacen las universidades para transferir conocimiento es a través del canal tradicional. Se evidencia que los investigadores que han publicado más artículos y libros tienen más disponibilidad para realizar actividades de transferencia de tecnología. Este canal depende, más que todo, del factor individual de transferencia de tecnología por parte del investigador (Aceytuno \& Sánchez-López, 2014). Se debe a su personalidad, habilidades, trayectoria, conexión con personas emprendedoras e interés por dedicarse a la investigación (Aceytuno \& Sánchez-López, 2014). La socialización de los resultados de investigación hace que el conocimiento sea aprovechable por la comunidad.

En el canal comercial, la creación de spin off ${ }^{4}$ y las licencias de patentes ${ }^{5}$ son los elementos más estudiados de la transferencia tecnológica y son la vía de comercialización de investigación universitaria (Aceytuno \& Sánchez-López, 2014). Desde las universidades, la generación de spin off contribuye, a través de incentivos económicos como resultado de la comercialización, a poder autofinanciar las investigaciones y a seguir creado más invenciones que favorezcan a investigadores, docentes y estudiantes. Asimismo, las patentes facilitan la protección del trabajo que se realizó en la investigación.

El canal bidireccional se da con relaciones de doble vía, en donde las partes se ponen de acuerdo para desarrollar proyectos conjuntos de $\mathrm{I}+\mathrm{D}+\mathrm{i}$. El canal servicios se puede determinar cuando una empresa o el gobierno contratan los servicios de consultoría para apropiarse de la información desarrollada en la universidad.

4 Un spin off es cualquier proceso de creación de empresa en el que unos individuos abandonan su organización original y crean una nueva empresa (Pirnay et al., 2003: 356, citado en Aceytuno \& Cáceres, 2009). Las empresas derivadas del spin off son creadas a partir de los resultados de investigación como semillas empresariales y tienen potenciales de innovación en el conocimiento tácito económicamente explotable, dado desde las universidades y los centros de investigación (Karnani, 2013). El investigador crea una empresa para explotar la tecnología y hay un actor que invierte para lograr esto.

5 Una patente es un derecho de exclusividad sobre una invención. El titular decide si la invención puede ser utilizada por terceros y de qué manera (OMPI, 2014). La patente es importante porque garantiza los derechos de propiedad y ayuda a establecer a quien se transfiere la invención. 
Leonardo Andrés Briceño Marín, María Eugenia Morales Rubiano Desafíos de la política pública colombiana frente a la transferencia de resultados de investigación universitaria

\section{Sistema Nacional de INNOVACión Y MEdios O CANALES DE TRANSFERENCIA}

\section{1. Ámbito internacional}

Las universidades con sus actividades de investigación básica son pilares de los SNI. En las universidades de América Latina es en donde se brinda más empleo a investigadores y reciben más apoyo financiero público para investigación (OCDE, 2009, citado en Calderón-Martínez \& García-Quevedo, 2013). En América Latina, las universidades son el actor que fomenta más la investigación, dadas las características de la tercera misión, en la cual no solo se enfocan en investigación y docencia, sino también enmarcan la transferencia y emprendimiento hacia la sociedad.

En Latinoamérica, se han propuesto reformas institucionales para incentivar la asignación de recursos y fortalecer la relación universidad-empresa (García, 2011). Esto se debe a que los canales de transferencia son débiles y necesitan impulsarse. Las universidades latinoamericanas tradicionalmente han tenido un papel más social relacionado con las necesidades de la región; para ello, la extensión ha sido una actividad académica fundamental (Calderón-Martínez \& García-Quevedo, 2013). Los contratos de la universidad con las entidades gubernamentales territoriales son principalmente para desarrollar proyectos de impacto social.

En Estados Unidos, las patentes académicas son de características institucionales, debido a la especialización y a la inversión y calidad en I + D apoyada con oficinas de transferencia de tecnología (Calderón-Martínez \& García-Quevedo, 2013).

En el contexto europeo, la política de innovación se fundamenta en la transferencia de conocimiento desde la universidad hacia la empresa. Por ejemplo, en Andalucía, España, las spin off desarrolladas a partir de resultados de investigación universitaria son potenciadoras de desarrollo económico y científico (Martínez \& Miranda, 2014). Se observa que en España las spin off son medios característicos de transferencia universitaria. 
En Europa se trabaja fuertemente para fortalecer la innovación tecnológica en las empresas a partir de la transferencia del conocimiento desde las universidades con los canales de centros tecnológicos, los organismos de investigación, las empresas de base tecnológica y otros. Así se busca lograr un crecimiento económico sostenible (Melero et al., 2011). En el Reino Unido, la universidad incorpora en el contenido curricular comportamientos empresariales y se vincula con la sociedad a través de un modelo educativo de aprendizaje dentro del sector productivo (Gibb, 1993, citado en Calderón y García, 2013).

Asimismo, en Asia Oriental hay un rápido desarrollo industrial a través de transferencia de conocimiento a las empresas con los canales de redes nacionales y regionales por empresas con el propósito de mejorar la competitividad (Machikita \& Ueki, 2013).

Sintetizando la revisión de las políticas públicas de $\mathrm{C}$ y $\mathrm{T}$ del ámbito internacional: los canales de transferencia poseen características relevantes. En América Latina, se busca fortalecer la relación universidad-empresa. En Europa, existe una fuerte relación entre universidad-empresa. Allí, los consorcios de transferencia de tecnología son de carácter transnacional. En Europa también se han consolidado redes de cooperación, se favorece la comercialización, la transferencia de tecnología, conferencias, publicaciones, consultorías y se tienen programas de intercambios internacionales de investigadores. En Asia, existen vínculos entre universidadempresa para la comercialización y una internacionalización de la $\mathrm{C}$ y la $\mathrm{T}$.

\section{2. Ámbito nacional}

El Sistema Nacional de Ciencia y Tecnología (SNCyT) está conformado por órganos y actores. El gobierno, la academia y el sector productivo participan en la formulación de políticas públicas y programas que incentiven la i+T. Se establece como una acción de la política nacional de fomento a la investigación y la innovación, apoyando a las universidades y centros de investigación a través de la financiación de proyectos (Colciencias \& Consejo Nacional de Ciencia y Tecnología (CNCyT), 2008). 
Leonardo Andrés Briceño Marín, María Eugenia Morales Rubiano Desafíos de la política pública colombiana frente a la transferencia de resultados de investigación universitaria

Las universidades del país y el SNCyT son actores que tienen un rol de generación, apropiación y transferencia de conocimiento para satisfacer las necesidades de la sociedad y se relacionan con otros actores coordinándose para lograr resultados (Llanos \& Dager, 2003). Por lo tanto, las universidades se especializan en la creación de conocimiento, mientras el sector productivo busca adquirir lo generado en las universidades para comercializarlo, lo cual permite contribuir a la dinamización de la investigación.

El SNCyT ha evolucionado adicionando el pilar de la innovación. Ahora se le denomina Sistema Nacional de Ciencia, Tecnología e Innovación (SNCTI). El SNCTI promueve las actividades de CyT y de $\mathrm{I}+\mathrm{D}+\mathrm{i}$ y fomenta los centros de investigación, parques tecnológicos, centros de productividad, incubadoras, empresas de base tecnológica, redes y elementos del Sistema. También, el SNCTI cuenta con programas, actividades de CyT y estrategias de instituciones públicas y privadas (Colombia, Congreso Nacional de la República, 2009).

En el SNCTI, además de Colciencias, también participan instituciones ejecutoras, definidoras e implementadoras de políticas de $\mathrm{Cy} \mathrm{T}+\mathrm{i}$, organismos de cooperación y relacionados. Esto es fundamental para el desarrollo de la transferencia de investigación de las universidades, ya que todos pueden interactuar en el Sistema.

A través de las instituciones del Estado, en función de un marco legal y de políticas económicas, se configuran las reglas del juego para la interacción de los actores de los sistemas de innovación. Su deber es regular la interacción, facilitar la transmisión de información y fortalecer la relación (Muñoz, 2005).

La capacidad jurídica adecuada de un país ayuda a promover la participación en los esquemas de colaboración para el licenciamiento de patentes, teniendo en cuenta la relación universidad-empresa-Estado (Corredor, 2012). Las políticas públicas en materia de $\mathrm{C}$ y $\mathrm{T}$ brindan a los actores una oportunidad de participación para concretar estrategias conjuntas. El SNCTI es la mejor manera para fortificar los canales. 
En la revisión bibliográfica, se identificó que las políticas públicas de $\mathrm{C}$ y $\mathrm{T}$ de Colombia tienen como objetivo incentivar los canales de transferencia mediante: (a) el fortalecimiento de la investigación en las regiones, a través de la integración territorial con los Comités Universidad-Empresa-Estado (CUEE); (b) trabajo colaborativo; (c) creación de redes para divulgar el conocimiento; (d) apropiación social del conocimiento en la población a través de la participación, articular actores públicos y privados, establecimiento de lineamientos de propiedad intelectual, incubación de empresas y (e) creación de oficinas de transferencia. Las relaciones interinstitucionales e interactivas se dan a través los Comités Universidad-EmpresaEstado, el SNCTI y los Clúters como Parquesoft y Guatiguará.

Los medios de transferencia de investigación universitaria en Colombia se pueden dar a través de spin off, relación universidad-empresa-Estado, apropiación y transferencia de patentes, contratos con entidades públicas y privadas, entre otros. A su vez, las políticas de $\mathrm{C}$ y $\mathrm{T}$ demuestran un esfuerzo por fomentar los canales de trasferencia. Pero el grado de desarrollo es bajo. Esto se demuestra por el número de patentes registradas por la Superintendencia de Industria y Comercio en comparación con países como Estados Unidos y China.

Igualmente, se presenta dificultad en las relaciones universidad-empresa por las limitaciones de recursos que tienen ambos actores y por la baja propensión a relacionarse (Rodríguez, Gómez, \& Valencia, 2013). Es necesario materializar las políticas en la realidad de los actores e invertir más en $\mathrm{I}+\mathrm{D}+\mathrm{i}$. Sin embargo, se destacan casos de éxito realizados en alianzas universidad-empresa-Estado de promoción de negocios innovadores. Por ejemplo, en Medellín se ha desarrollado satisfactoriamente la Ruta $\mathrm{N}$ y Tecnnova, la cual apoya planes de negocio para spin off de Antioquia. Secopind es un esquema de asociación con los actores mencionados, adicionando el apoyo de la Organización Mundial de la Propiedad Intelectual y la Superintendencia de Industria y Comercio, como red para promover el licenciamiento y comercialización de propiedad intelectual (Gómez \& Mitchell, 2014). 


\section{EL PAPEL DEL GOBIERNO Y LAS POLÍTICAS PÚBLICAS DE FOMENTO A LA TRANSFERENCIA DE RESULTADOS DE INVESTIGACIÓN UNIVERSITARIA}

Las políticas públicas son configuraciones entre actores que se diseñan para el bien común y para la construcción de una imagen de la realidad a la cual se pretende llegar (Muller, 2006, citado en Perdomo, Arias, \& Lozada, 2013). Entonces, las políticas públicas son construidas para satisfacer las necesidades de la sociedad y de los grupos de interés.

Los elementos centrales de las políticas públicas son: implicaciones del gobierno, la existencia de una problemática, la definición de los procesos y los objetivos (Roth, 2002). Este estudio, por ejemplo, demuestra y enfatiza que existe una problemática relacionada con la promoción de la transferencia de resultados investigación universitaria hacia el entorno. Esto se realiza con el fin de que el conocimiento desarrollado no se quede en las aulas, sino que se pueda explotar comercialmente y beneficiar a la sociedad.

El Estado puede intervenir con instrumentos de incentivos que no obliguen a los actores objetivos a ciertos comportamientos, sino que dirijan la conducta a través de la recompensa (Roth, 2002). Las políticas de C y T pueden estar diseñadas, en este sentido, en la reducción de tributos, financiación, otorgación de becas para maestría y doctorado, apoyo en incubación de empresas. Así, las universidades, investigadores y empresas pueden promover el desarrollo de $\mathrm{I}+\mathrm{D}+\mathrm{i}$.

La implementación de políticas públicas se realiza mediante acciones de individuos o grupos públicos o privados para realizar objetivos anteriormente pactados (Aguilar, 1993). La participación social ayuda a concertar objetivos de promoción de C y T. Por ejemplo: establecer reuniones con los representantes de los sectores en las que todos puedan exponer sus necesidades y dificultades. 


\section{Políticas públicas de C y T en el Ámbito internacional}

\section{Región americana}

Las políticas públicas existen cuando las instituciones del Estado, se comprometan a cumplir un objetivo derivado de una problemática (Roth, 2002). El Estado debe tener como objetivo la competitividad, la cual puede ser incentivada por medio de la innovación. Un país innovador no es dependiente de otro, como tradicionalmente se ve en mayor parte en Latinoamérica. En esas áreas latinoamericanas, desde la sustitución de importaciones, se propuso la meta de industrializase en materia de tecnología, capital y escala, es decir, a segmentos cada vez más exigentes (Bielschowsky, 2006). En general, las políticas de $\mathrm{C}$ y $\mathrm{T}$ en Latinoamérica van orientadas a la promoción, a través de la inversión extranjera directa (Gómez \& Mitchell, 2014). La mayor producción de los países latinoamericanos se basa en materias primas, las cuales no tienen valor agregado. Debido a esa situación, los países industrializados tienen más ventajas en el desarrollo económico.

En el Perú, es necesario incrementar la inversión en $\mathrm{I}+\mathrm{D}+\mathrm{i}$ y promover la relación universidad empresa a través de políticas públicas (UNCTAD, 2012). Se puede inferir que este país también tiene dificultades igual que Colombia para la financiación de la $\mathrm{C}$ y la $\mathrm{T}$. Por consiguiente, se debe hacer énfasis en este aspecto en ambos países.

Las políticas públicas implantadas en México entre los años 1994 y 2000 estaban enfocadas en cubrir necesidades del desarrollo empresarial a través de la búsqueda de eficiencia de los recursos de la nación. Esos recursos iban a ser destinados a la I +D, a crear empresas y a vincularlas al sector educativo. Entre 2006 a 2012, se desarrolló la primera Ley de Ciencia y Tecnología, la cual regulaba el soporte del Gobierno la I de $\mathrm{C}$ y $\mathrm{T}$. Esta ley se basaba en relacionar la I con la competitividad para promover la economía local. Entre 2012 a 2018, se ha buscado propiciar la transferencia de conocimiento de las universidades, el emprendimiento y la propiedad industrial (Vizcarra, Torres, \& Ruiz, 2014).

De modo similar, el plan estratégico de República Dominicana de C+T+i 20082018 busca consolidar la oferta científica y tecnológica de las empresas, fomentar 
Leonardo Andrés Briceño Marín, María Eugenia Morales Rubiano Desafíos de la política pública colombiana frente a la transferencia de resultados de investigación universitaria

la relación universidad-empresa, crear incubación de empresas y establecer redes de investigación (UNCTAD, 2012). De esta forma, se da relevancia a la relación de los actores como mecanismo de transferencia a través del emprendimiento.

Los Estados Unidos de América han dominado el desarrollo de C y T históricamente, debido a su gobernanza, a la transparencia de sus instituciones, a su sistema pluralista y, sobre todo, a que el desarrollo científico en ese país se concibe como una filosofía (Parthasarathy, 2008). En Estados Unidos, se publicitan los procesos tecnológicos como parte de la cultura del país y se deja participar a los actores. Los cluster son potenciadores de la Cy la T. Ejemplos de esos clusters son: Silicón Valley en California, optoelectrónica en Rochester y bioinformática en Boston. Adicionalmente, los estadounidenses tienen una fuerte cultura de innovación, aceptación al riesgo y eficacia empresarial (Gómez \& Mitchell, 2014).

\section{Región europea}

Actualmente, se promociona la transferencia de tecnología entre la universidad y el sector productivo para contribuir al desarrollo económico y social. La Unión Europea (UE) tiene políticas de proporcionalidad que dan participación a distintos actores y contribuyen a generar herramientas de eficiencia y calidad para la sociedad del conocimiento. La UE promueve la creación de consorcios trasnacionales para impulsar la C y la T (Pestańa, 2013). En materia de cooperación, los actores se relacionan internacionalmente mediante de bloques en pro de ser más competitivos.

En España, se implementaron políticas que dan incentivos económicos en la creación de empresas por transferencia a través de la Ley Orgánica de Universidades, promulgada en el año 2001 y reformada en el año 2007. Esta ley favorece la comercialización y transferencia (Aceytuno \& Sánchez-López, 2014). En la región española, se conoce la importancia de impulsar la transferencia universitaria.

Desde el año 2000, el desarrollo empresarial y la innovación han alcanzado un punto óptimo en España. Esto se ha alcanzado a través de las políticas públicas que permiten que los actores privados participen y se relacionen con el Estado en 
el diseño y ejecución de las políticas. Con la Ley 14 de 2011, el gobierno creó la Agencia Estatal de Investigación para impulsar la investigación científica a través de la relación institucional entre innovación y competitividad (Perdomo et al., 2013).

Las universidades españolas impulsan el avance del conocimiento científico y son actrices en la economía de los países, puesto que, a través de la innovación, se crean nuevos empleos y se aumentan los ingresos de las naciones (Aceytuno \& SánchezLópez, 2014). El sector productivo y el gobierno deben brindar ayuda y financiación a las universidades por su contribución al desarrollo.

Actualmente, la administración pública española se ha esforzado por impulsar la creación de spin off, las cuales son utilizadas como medio de transferencia de conocimiento. A través de sus actividades, estas spin off generan recursos para su autosostenimiento (Martínez \& Miranda, 2014). No solo es importante otorgar recursos a las universidades para $\mathrm{I}+\mathrm{D}+\mathrm{i}$ y para generar emprendimiento, sino que también las políticas públicas se enfoquen en crear mecanismos de eficiencia administrativa empresarial en las incubaciones.

Las leyes en Alemania y en Suecia promueven que las universidades investiguen e interactúen con el entorno. Esto se puede dar, por ejemplo, mediante conferencias, publicaciones, consultoría en sector público y transferencia de tecnología. Los investigadores que reciben el apoyo de la infraestructura de transferencia tienen más propensión a patentar. Los honorarios por los libros publicados y conferencias también facilitan el incremento de transferencia en estos dos países (Sellenthin, 2009).

Alemania tiene un sistema educativo de alta calidad y vincula claramente la industria y los centros de investigación (Gómez \& Mitchell, 2014). Del mismo modo, Alemania destina gran parte de su presupuesto a la investigación. Para aprovechar el conocimiento global, Alemania atrae a investigadores talentosos de todo el mundo a través de programas de intercambio como el Servicio Alemán de Intercambio Académico (DAAD).

Los países europeos son conscientes de la importancia de la definición de los sistemas nacionales en términos de calidad y eficiencia para adentrarse a la sociedad del 
conocimiento. En cuanto al Reino Unido, por ejemplo, se plantea una división entre enseñanza y la investigación. Allá, se da más peso a la investigación, lo cual se refleja en la recompensa de beneficios (Kindelán, 2013). La investigación en un país es fuente de empleo, competitividad, desarrollo y entre otros beneficios.

Para fomentar la $\mathrm{C}$ y la $\mathrm{T}$, además de incrementar la financiación, también se debe dar exención tributaria, proteger la propiedad intelectual y fortalecer la corporación, la universidad y las redes de cooperación (Ramos, 2006). La formulación, implementación y ejecución debe ser integral y dar peso a todos los factores que promuevan la $\mathrm{I}+\mathrm{D}+\mathrm{i}$.

Algunos casos de éxito se deben a acciones concretas. En Finlandia, el sistema es simple, transparente e independiente de instituciones financieras. En Irlanda, ha habido proactividad en asignación de recursos públicos y se estableció un compromiso a largo plazo. En Nueva Zelanda, se enfocan los recursos para mejorar la educación y se otorga el liderazgo de innovación a las empresas (Gómez \& Mitchell, 2014).

\section{Región asiática}

Desde 1980, la C y la T se han convertido en la principal fuerza productiva de China y se ha notado su avance tecnológico. Desde ese entonces, el gobierno promueve políticas de $\mathrm{I}+\mathrm{D}$ que brindan financiación y mayor autonomía a los centros de investigación con el objetivo de que las empresas participen en I+D. La reforma de $\mathrm{C}$ y $\mathrm{T}$ ha sido eficaz para motivar a las universidades a contribuir a las capacidades innovadoras en las empresas y promover los vínculos (Xiwei \& Xiangdong, 2007). El programa Torch desarrolla industrias de alta tecnología a través de parques industriales y de software, así como clúster de alto impacto (Gómez \& Mitchell, 2014). Adicionalmente, China tiene una ventaja en términos de competitividad: costos de producción tan bajos que casi ningún país los puede igualar.

La transferencia de tecnología en China tiene un papel clave en el desarrollo de las invenciones, las cuales son llevadas al mercado internacional. Se desarrolla a través de un sistema de transferencia en el que se adopta la tecnología en los mercados 
y las industrias. Además, con apoyo del entorno político y jurídico se modifican los requisitos para que los actores puedan participar en dicha transferencia (Gross, 2013). El apoyo del gobierno con la internacionalización de los resultados de la investigación promueve la obtención de recursos financieros a mayor nivel en pro de poder seguir innovando.

Las políticas de $\mathrm{C}$ y $\mathrm{T}$ deben estar alineadas, integradas y sincronizadas para reducir las brechas en los avances tecnológicos. Las medidas adoptadas por China, en cuanto a I+D, sirven para potencializar el crecimiento económico (Chen-Chen, Pei-Lee, Ooi, \& Kim-Lan, 2009). Debido a los avances rápidos en la tecnología, es muy difícil competir globalmente.

Los países asiáticos se han preparado para competir a nivel mundial. Japón, con el Plan Básico de C y T 2002-2006, incrementó la financiación en la productividad y fomentó las relaciones entre universidad y la corporación con el propósito de facilitar la comercialización (Ramos, 2006). Por otra parte, Corea, tiene objetivos de C y $\mathrm{T}$ a largo plazo, da importancia a la $\mathrm{I}+\mathrm{D}+\mathrm{i}$ y a la calidad de la educación para el desarrollo del país. Sus empresas de tecnología son de alcance internacional (Gómez $\&$ Mitchell, 2014). Otros países competitivos, como Singapur, se caracterizan por tener transparencia en los procesos y en los resultados. Por ello, cuentan con amplia efectividad.

\section{ÁMBITo NACIONAL}

\section{Leyes en el contexto actual}

Con la Ley 29 de 1990 y su modificación en la Ley 1286 de 2009, la cual transforma a Colciencias en un Departamento Administrativo, se ha buscado incentivar la investigación e incubación de empresas en Colombia (Charry \& Pérez, 2013). En la Ley 29 de 1990, se puede notar el interés del Estado en promover la C y la T a través de la creación de fomento investigativo, adopción técnicas de investigación de nivel mundial y asignación de recursos (Congreso Nacional de la República, 1990). 
Leonardo Andrés Briceño Marín, María Eugenia Morales Rubiano Desafíos de la política pública colombiana frente a la transferencia de resultados de investigación universitaria

La Ley 1286 de 2009 tiene como objetivo fortalecer a Colciencias y la investigación científica. Además, respecto a la transferencia de investigación universitaria, en esa ley se puede encontrar marcos para la apropiación y divulgación del conocimiento para ser más competitivos. Los agentes que giran en entorno a la investigación pueden desarrollar redes de investigación e incubadoras de empresas. Todo lo anterior apunta promover el desarrollo de la población de Colombia (Congreso Nacional de la República, 2009). Por su parte, en el plan estratégico de Colciencias, se define que esta entidad es quien diseña, evalúa, ejecuta y coordina la política nacional de C+T+I (Colciencias, 2011). A través de plataformas regionales y redes de apoyo a la innovación, se busca impulsar la relación Universidad-Empresa-Estado (Colciencias, 2011). Entonces, se Colciencias desempeña el rol de formular e implementar políticas que incentiven la $\mathrm{C}$ y la $\mathrm{T}$ en Colombia y busca integrar a todos los actores.

Por otra parte, existen las regalías, las cuales son compensaciones económicas por explotar un recurso no renovable. Según determina la ley colombiana, esas económicas van dirigidas al Fondo Nacional de Regalías, a los departamentos y a los municipios (Asamblea Nacional Constituyente, 1991). Del Sistema General de Regalías, se destinan recursos para el Fondo de $\mathrm{C}$ y $\mathrm{T}+\mathrm{i}$ que van más enfocados financiar los proyectos territoriales de las regiones. En dichos proyectos, interactúan los actores del Modelo Triple Hélice y el Triángulo de Sábato.

Recientemente, se aumentaron significativamente los ingresos para $\mathrm{C}$ y $\mathrm{T}+\mathrm{i}$ en el Sistema General de Regalías (OCDE, 2014). En la Ley 1530 de 2012, se establece la regulación y el funcionamiento de este Sistema. Dicha ley determina unas funciones adicionales de Colciencias: ejercer la secretaria técnica del Órgano colegiado de administración y decisión y brindar los recursos humanos y la infraestructura necesarios para tal fin. Colciencias propone metodologías para el manejo de los proyectos que se van a financiar y verifica el cumplimiento de los mismos con el Fondo de Ciencia, Tecnología e Innovación. Este fondo busca mejorar la capacidad de C y T+i y la competitividad regional a través de apropiación del conocimiento de la población. Los requisitos son establecidos por la Comisión Rectora, la cual es un órgano que define la política general del Sistema General de Regalías en Colombia (Congreso Nacional de la República, 2012). 
En cuanto a la generación de empresas, se da una normativa con la Ley de emprendimiento (Ley 1014 de 2006). Esa ley dictamina que en la academia se debe ofrecer una cátedra de emprendimiento para fomentar el espíritu emprendedor. Además, mediante esa ley se crea una Red Nacional y una Red Regional de emprendimiento (Gómez \& Mitchell, 2014).

\section{Plan Nacional de Desarrollo 2010-2014}

El Plan Nacional de Desarrollo establece que la innovación es clave para ser más competitivo en la producción y, además, involucra a actores públicos y privados para el progreso social (Departamento Nacional de Planeación, 2011). Igualmente, reconoce la importancia de la innovación para el desarrollo social y económico del país (OCDE, 2014). Es necesario dar valor agregado a la producción nacional a través de la $\mathrm{C}$ y la $\mathrm{T}$, no solo producir materias primas.

Con las "locomotoras de desarrollo", se busca el desarrollo social y económico a través de la innovación para incorporar la tecnología en el sector productivo (Colciencias, 2013). Para lograr esto, se debe fortalecer la relación universidadempresa mediante lineamientos de la propiedad intelectual y el emprendimiento universitario (Departamento Nacional de Planeación, 2009). La universidad como generadora de conocimiento es un actor al cual se deben destinar recursos y darle beneficios por sus resultados de investigación.

\section{Políticas y estrategias}

A través de la Política Nacional de Ciencia, Tecnología e Innovación, se apoya financieramente a las universidades y los centros de investigación. Para fortalecer la investigación en las regiones, se adquieren de equipos tecnológicos (Departamento Nacional de Planeación, 2009). Las estrategias para destinar recursos para C y T son lo más efectivo para impulsar la transferencia, ya que es muy costoso desarrollar proyectos de investigación; más aún si son de ciencias puras o ingenierías. 
Leonardo Andrés Briceño Marín, María Eugenia Morales Rubiano Desafíos de la política pública colombiana frente a la transferencia de resultados de investigación universitaria

Entre las estrategias de la Política Nacional de Fomento a la Investigación y la Innovación, está el apoyo a la formación avanzada de investigadores, a las universidades y centros de investigación y el fomento a la apropiación del conocimiento y las capacidades regionales (Colciencias \& Consejo Nacional de Ciencia Y Tecnología (CNCyT), 2008).

Con la Estrategia Nacional de Innovación, se fortalece el crecimiento por medio de financiación con recursos de la nación y regalías para la creación de oficinas de transferencia de tecnología. Así, el conocimiento de las universidades se puede explotar comercialmente. En los proyectos de universidad-empresa, se financian proyectos para transferir conocimiento al sector productivo mediante el trabajo colaborativo y recursos de la nación (Departamento Nacional de Planeación, 2011). La inversión del gobierno en investigación es fundamental para que la tecnología de un país avance (Laredo, 2007). A través de financiación, también se pretende hallar todos los mecanismos para incentivar la $\mathrm{I}+\mathrm{D}+\mathrm{i}$ y reducir la brecha existente en la destinación de recursos en actividades de $\mathrm{C}$ y $\mathrm{T}$.

La Apropiación Social del Conocimiento es una estrategia que busca relacionar la tecnología y la sociedad. Esta relación se da entorno a los actores generadores de conocimiento, en unión con la sociedad civil, a través de las siguientes líneas de acción: transferencia e intercambio de conocimiento, participación ciudadana, comunicación y gestión del conocimiento. A su vez, la Política Nacional de Apropiación Social de la $\mathrm{C}+\mathrm{T}+\mathrm{i}$ aspira a que la sociedad colombiana, en general, se apropie del tema (Colciencias, 2012). Esta política da oportunidades de participación general, en términos de políticas públicas de conocimiento. Por ende, estas políticas son de interés común y añaden la sociedad civil a los actores tradicionales (universidad-empresa-Estado).

Un objetivo específico de la Estrategia Nacional para Apropiación Social de la Ciencia, Tecnología e Innovación es dar participación ciudadana en la toma de decisiones de políticas públicas relacionadas (Colciencias, 2010). Así, se articula el concepto de innovación social con la Política Nacional de Ciencia, Tecnología e Innovación (Departamento Nacional de Planeación, Colciencias \& ANSPE, 2013). La participación de la sociedad en las políticas de C y $\mathrm{T}$ y la búsqueda de desarrollo 
social, a través de la innovación, mejora la integración de los factores políticos con todos los actores.

En cuanto a transferencia de resultados de investigación universitaria, en la estrategia mencionada anteriormente se destacan: la inversión en educación superior, la inserción de doctores en el sector productivo, la financiación de proyectos de universidad- empresa, la creación de oficinas de transferencia y las deducciones tributarias (Departamento Nacional de Planeación, 2011). Mediante la unión de todos esos factores y con base en los objetivos de las políticas de $\mathrm{C}$ y $\mathrm{T}$, se busca impulsar la capacidad de innovación del país y del sector productivo.

En el documento Colombia Visión 2019, se proponen estrategias para superar la escaza capacidad de desarrollo de $\mathrm{C}$ y $\mathrm{T}$. La línea de acción principal es el apoyo a las investigaciones de $\mathrm{C}+\mathrm{T}+\mathrm{i}$ en la educación y las instituciones. También, se apunta al fortalecimiento de las relaciones entre empresas y universidades, al fomento a la transferencia de tecnología, a la promoción de la agenda pública de la investigación científica, entre otros (Departamento Nacional de Planeación \& Colciencias, 2006). Adicionalmente, se asevera que la financiación de $\mathrm{I}+\mathrm{D}+\mathrm{i}$ es muy baja, en comparación a los estándares internacionales. Por consiguiente, se determina que es necesario incrementar dicha financiación por medio de la relación universidad-empresa-Estado.

De otra parte, la educación inclusiva procura promover el desarrollo investigativo y fomentar la colaboración entre instituciones de educación superior con (Ministerio de Educación Nacional, 2013). Entonces, se plantea que, además de la relación entre los actores, debe haber alianzas entre las universidades para el desarrollo científico con la sociedad.

Asimismo, se insiste en que se deben incrementar los vínculos entre universidades para crear redes de investigación conformadas por regiones y en que la investigación debe relacionarse mediante centros universidad-empresa para fortalecer la productividad empresarial (CESU, 2013). Con eso, se busca lograr una integración territorial a través de los Comités Universidad-Empresa-Estado (CUEE), los cuales son instancias regionales de acuerdos entre el sector productivo y las universidades. Los CUEE se conforman mediante grupos de investigación para contribuir a establecer proyectos 
óptimos para el impulso productivo (Ministerio de Educación Nacional, 2009). Los comités de las regiones se caracterizan por compartir rasgos similares culturales, sociales y económicos. Gracias a su compatibilidad, la integración regional entre los actores de la $\mathrm{C}$ y la $\mathrm{T}$ facilitan más los avances.

A continuación, en la tabla 2, se muestra una síntesis de las normas, las políticas, los planes y las estrategias que el gobierno nacional y otros actores del SNI han generado con el objetivo de fomentar los procesos de transferencia de conocimiento e innovación.

Tabla 2. Muestra histórica de políticas relacionadas con $\mathrm{C}$ y $\mathrm{T}$ en Colombia

\begin{tabular}{|c|c|c|c|c|}
\hline Tipo & Nombre & Ańo & $\begin{array}{l}\text { Contribución a los procesos } \\
\text { de transferencia de } \\
\text { conocimiento e innovación }\end{array}$ & Origen \\
\hline \multirow{6}{*}{ Norma } & $\begin{array}{l}\text { Constitución Política } \\
\text { de Colombia }\end{array}$ & 1991 & $\begin{array}{l}\text { Autonomía universitaria, } \\
\text { fomento de investigación, y } \\
\text { determinación de regalías }\end{array}$ & $\begin{array}{l}\text { Asamblea } \\
\text { Nacional } \\
\text { Constituyente }\end{array}$ \\
\hline & Ley 29 de 1990 & 1990 & $\begin{array}{l}\text { Promulgación de Ley de } \\
\text { ciencia y tecnología CyT, } \\
\text { institucionalización del } \\
\text { Sistema Nacional de Ciencia y } \\
\text { Tecnología, SNCyT }\end{array}$ & $\begin{array}{l}\text { Congreso de } \\
\text { la República } \\
\text { Colombia }\end{array}$ \\
\hline & Ley 6 de 1992 & 1992 & Incentivos tributarios a la $\mathrm{Cy} T$ & \\
\hline & Ley 344 de 1996 & 1996 & $\begin{array}{l}\text { Recursos SENA a Programas } \\
\text { de Competitividad y Desarrollo } \\
\text { Tecnológico Productivo }\end{array}$ & \\
\hline & Ley 1014 de 2006 & 2006 & Ley de emprendimiento & \\
\hline & Ley 1286 de 2009 & 2009 & $\begin{array}{l}\text { Colciencias se convierte en un } \\
\text { Departamento Administrativo, } \\
\text { creación del Sistema Nacional } \\
\text { de Ciencia, Tecnología e } \\
\text { Innovación (SNCTI) }\end{array}$ & \\
\hline
\end{tabular}




\begin{tabular}{|c|c|c|c|c|}
\hline Tipo & Nombre & Año & $\begin{array}{l}\text { Contribución a los procesos } \\
\text { de transferencia de } \\
\text { conocimiento e innovación }\end{array}$ & Origen \\
\hline \multirow{3}{*}{ Norma } & Ley 1530 de 2012 & 2012 & $\begin{array}{l}\text { Regulación y el funcionamiento } \\
\text { del Sistema General de Regalías }\end{array}$ & \\
\hline & Decreto 585 de 1991 & 1991 & $\begin{array}{l}\text { Creación del Consejo Nacional } \\
\text { de Ciencia y Tecnología } \\
(\mathrm{CNCyT}) \text {,creación de las } \\
\text { Comisiones Regionales } \\
\text { de Ciencia y Tecnología, } \\
\text { adscripción de Colciencias al } \\
\text { Departamento Nacional de } \\
\text { Planeación (DNP) }\end{array}$ & $\begin{array}{l}\text { Ministro de } \\
\text { Gobierno de la } \\
\text { República de } \\
\text { Colombia. }\end{array}$ \\
\hline & $\begin{array}{l}\text { Decreto } 2828 \text { de } \\
2006\end{array}$ & 2006 & $\begin{array}{l}\text { Organización del Sistema } \\
\text { Administrativo Nacional de } \\
\text { Competitividad }\end{array}$ & $\begin{array}{l}\text { Presidente de } \\
\text { la República de } \\
\text { Colombia }\end{array}$ \\
\hline \multirow{6}{*}{ Política } & $\begin{array}{l}\text { Conpes } 2739 \text { de } \\
1994\end{array}$ & 1994 & Primer Conpes de $\mathrm{C}$ y $\mathrm{T}$ & $\begin{array}{l}\text { Departamento } \\
\text { Nacional de } \\
\text { Planeación } \\
\text { (DNP) }\end{array}$ \\
\hline & $\begin{array}{l}\text { Conpes } 3080 \text { de } \\
2000\end{array}$ & 2000 & $\begin{array}{l}\text { Política de Ciencia y } \\
\text { Tecnología }\end{array}$ & \\
\hline & $\begin{array}{l}\text { Conpes } 3439 \text { de } \\
2006\end{array}$ & 2006 & $\begin{array}{l}\text { Principios de competitividad y } \\
\text { productividad }\end{array}$ & \\
\hline & $\begin{array}{l}\text { Conpes } 3527 \text { de } \\
2008\end{array}$ & 2008 & $\begin{array}{l}\text { Política Nacional de } \\
\text { Competitividad y } \\
\text { Productividad Visión } 2032\end{array}$ & \\
\hline & $\begin{array}{l}\text { Conpes } 3582 \text { de } \\
2009\end{array}$ & 2009 & Política nacional de $\mathrm{C}+\mathrm{T}+\mathrm{i}$. & \\
\hline & $\begin{array}{l}\text { Conpes } 3668 \text { de } \\
2010\end{array}$ & 2010 & $\begin{array}{l}\text { Informe de Seguimiento } \\
\text { a la Política Nacional } \\
\text { de Competitividad y } \\
\text { Productividad. Ley } 1253 \text { de } \\
2008\end{array}$ & \\
\hline
\end{tabular}




\begin{tabular}{|c|c|c|c|c|}
\hline Tipo & Nombre & Año & $\begin{array}{l}\text { Contribución a los procesos } \\
\text { de transferencia de } \\
\text { conocimiento e innovación }\end{array}$ & Origen \\
\hline \multirow{3}{*}{ Política } & $\begin{array}{l}\text { Conpes } 3637 \text { de } \\
2011\end{array}$ & 2011 & $\begin{array}{l}\text { Desarrollo comercial de la } \\
\text { biotecnología sostenible }\end{array}$ & \\
\hline & $\begin{array}{l}\text { Política Nacional de } \\
\text { Apropiación Social de } \\
\text { la C+T+i. }\end{array}$ & 2005 & $\begin{array}{l}\text { Inclusión a sociedad del } \\
\text { conocimiento }\end{array}$ & $\begin{array}{l}\text { Colciencias- } \\
\text { Consejo } \\
\text { Nacional de } \\
\text { Ciencia y } \\
\text { Tecnología } \\
\text { (CNCyT) }\end{array}$ \\
\hline & $\begin{array}{l}\text { Política nacional } \\
\text { de fomento a la } \\
\text { investigación y la } \\
\text { innovación. }\end{array}$ & 2008 & $\begin{array}{l}\text { Desarrollo a partir de la } \mathrm{Cy} \\
\text { la } \mathrm{T}\end{array}$ & $\begin{array}{l}\text { Colciencias- } \\
\mathrm{CNCyT}\end{array}$ \\
\hline \multirow{3}{*}{ Plan } & $\begin{array}{l}\text { Plan Nacional } \\
\text { de Desarrollo } \\
2010-2014\end{array}$ & 2010 & $\begin{array}{l}\text { Innovación como una de las } \\
\text { cinco "locomotoras" }\end{array}$ & DNP \\
\hline & $\begin{array}{l}\text { Plan Estratégico } \\
\text { Sectorial de } \\
\text { Colciencias } \\
2011-2014\end{array}$ & 2012 & $\begin{array}{l}\text { Plan Estratégico Sectorial de } \\
\mathrm{C}+\mathrm{T}+\mathrm{I} \text { a través de objetivos y } \\
\text { metas }\end{array}$ & Colciencias \\
\hline & $\begin{array}{l}\text { Plan Estratégico } \\
\text { Institucional } \\
\text { de Colciencias } \\
\text { 2011-2014 }\end{array}$ & 2014 & $\begin{array}{l}\text { Plan Estratégico del cuatrienio } \\
\text { con directrices del Plan } \\
\text { Nacional de Desarrollo } \\
2010-2014\end{array}$ & Colciencias \\
\hline \multirow{3}{*}{ Estrategia } & $\begin{array}{l}2019 \text { Visión } \\
\text { Colombia II } \\
\text { Centenario }\end{array}$ & 2006 & $\begin{array}{l}\text { Desarrollo social a través de } \\
C+T+i\end{array}$ & DNP \\
\hline & Visión 2032 & 2006 & $\begin{array}{l}\text { Colombia como uno de los } \\
\text { tres países más competitivos de } \\
\text { Latinoamérica }\end{array}$ & $\begin{array}{l}\text { Comisión } \\
\text { Nacional de } \\
\text { Competitividad }\end{array}$ \\
\hline & $\begin{array}{l}\text { Comités universidad- } \\
\text { empresa-estado- } \\
\text { CUEE. }\end{array}$ & 2007 & $\begin{array}{l}\text { Creación de instancias } \\
\text { regionales de relación } \\
\text { universidad-empresa-Estado }\end{array}$ & $\begin{array}{l}\text { Grupos de } \\
\text { investigación- } \\
\text { sector } \\
\text { productivo y } \\
\text { Gobierno }\end{array}$ \\
\hline
\end{tabular}




\begin{tabular}{|lllll}
\hline \multicolumn{1}{|c|}{ Tipo } & \multicolumn{1}{c}{ Nombre } & Año & $\begin{array}{c}\text { Contribución a los procesos } \\
\text { de transferencia de } \\
\text { conocimiento e innovación }\end{array}$ & Origen \\
\hline Estrategia & $\begin{array}{l}\text { Estrategia nacional de } \\
\text { apropiación social de } \\
\text { la } \mathrm{C}+\mathrm{T}+\mathrm{i}\end{array}$ & 2010 & $\begin{array}{l}\text { Apropiación social del } \\
\text { conocimiento con I+i para } \\
\text { desarrollo social y económico }\end{array}$ & Colciencias \\
$\begin{array}{l}\text { Estrategia Nacional } \\
\text { de Innovación. }\end{array}$ & 2011 & Potenciar innovación. & DNP
\end{tabular}

Nota: Tabla elaborada por los autores a partir de documentos descritos en esta tabla de DNP \& Colciencias, 2006.

Del análisis del apartado número 3 y de la vinculación a los elementos del "radar" de la tercera misión de la universidad citado por Laredo (2007), se identifican algunos posibles desafíos de la política pública para Colombia, los cuales involucran a los diferentes actores del SNI (ver la tabla 3).

Tabla 3. Desafíos de la política pública colombiana desde la tercera misión universidad

\begin{tabular}{|c|c|}
\hline Asunto & Desafíos \\
\hline Recursos humanos & Promover más estudiantes de doctorado. \\
\hline Propiedad intelectual & Crear programas de consultoría en patentes. \\
\hline Spin off & Exención tributaria y ayuda administrativa. \\
\hline Contratos con la industria & $\begin{array}{l}\text { Establecer vínculos formales de contratos con } \\
\text { empresas. }\end{array}$ \\
\hline Contratos con organismos públicos & $\begin{array}{l}\text { Vincular recién egresados a entidades públicas } \\
\text { para investigación. }\end{array}$ \\
\hline Diseño y evaluación en políticas públicas & $\begin{array}{l}\text { Generar y evaluar una agenda de participación } \\
\text { en construcción de políticas públicas. }\end{array}$ \\
\hline Participación en la vida social y la vida cultural & Crear programas de cultura de $\mathrm{I}+\mathrm{D}+\mathrm{i}$. \\
\hline Comprensión pública de ciencia & $\begin{array}{l}\text { Mejorar el nivel de educación profesional de la } \\
\text { población colombiana. }\end{array}$ \\
\hline
\end{tabular}

Nota: Tabla elaborada por los autores a partir de datos de OEU citado en Laredo, 2007 
Leonardo Andrés Briceño Marín, María Eugenia Morales Rubiano

\section{Algunas políticas de las universidades colombianas QUE BUSCAN GENERAR EL MARCO PARA LA TRANSFERENCIA.}

\subsection{Universidades públicas}

En la Universidad Nacional de Colombia, existe una política de liderazgo en investigación. Esa política se aleja del modelo de universidad de enseńanza y se enfoca en ser investigativa. Así, se busca aportar el conocimiento para el desarrollo de la sociedad. La Universidad Nacional cuenta con publicaciones en artículos de revistas indexadas nacionales e internacionales y amplia oferta de doctorados. Realiza la transferencia a través de grupos, centros, consorcios, redes y otros. En la División de Extensión, se resalta el Programa de Integración Regional e Integración Tecnológica, relacionado con megaproyectos de impacto regional, y el Programa de Apoyo a Iniciativas Universitarias, el cual se gestiona con entidades externas (Universidad Nacional de Colombia, 2014). En la sede Bogotá, se coordina una alianza de integración con varias universidades de Bogotá y empresas (Pineda et al., 2011). Además, la Universidad Nacional hace parte de Connect Bogotá Región. Asimismo, realiza Convocatorias de Gestión Tecnológica e Innovación en las que hay proyectos para desarrollar y unas propuesta de reglamentación y emprendimiento de base tecnológica para la transferencia (Bonilla, 2012). La Universidad también participa en convocatorias externas e internas de investigación.

El Acuerdo 035 de 2003 del Consejo Académico reglamenta la propiedad intelectual (PI) en la Universidad Nacional de Colombia. En dicho acuerdo, se definen los conceptos sobre PI, se establecen las reglas de juego y se resalta la distribución de porcentaje de regalías de acuerdo con la comercialización de la PI (Universidad Nacional de Colombia, 2005).

Por otra parte, en la Universidad Militar Nueva Granada (UMNG) existe una Vicerrectoría de Investigaciones. Una de las funciones de esa vicerrectoría es fomentar las relaciones interinstitucionales de $\mathrm{C}+\mathrm{T}+\mathrm{I}$ en el país y el exterior. Para ello, cuenta con una dependencia llamada División de Desarrollo Tecnológico e Innovación, la cual se relaciona con el entorno por medio de la transferencia tecnológica con el 
sector productivo, la protección mediante patentes y la búsqueda de incubación de empresas. En la UMNG, se fortaleció el emprendimiento universitario a través de la Resolución 3726 de 2013, la cual creó el Centro de Innovación y Emprendimiento Neogranadino (CIEN) para apoyar el emprendimiento innovador de la comunidad académica (Universidad Militar Nueva Granada, 2009). Esto demuestra que la UMNG tiene objetivos delimitados para transferir resultados de investigación.

El reglamento de PI de la UMNG es el Acuerdo 12 de 2013. Éste establece normas para las personas que pertenecen a la institución, define conceptos, y establece principios, derechos, deberes y las regalías sobre PI. En el Acuerdo 08 de 2013, se actualiza el Estatuto del Sistema de Ciencia, Tecnología e Innovación $(\mathrm{C}+\mathrm{T}+\mathrm{I})$ de la UMNG. Allí, define la estructura organizacional de $\mathrm{C}+\mathrm{T}+\mathrm{I}$, sus funciones y los lineamientos para incentivar la investigación científica y protección de la PI (Universidad Militar Nueva Granada, 2014).

En las convocatorias de la UMNG, se incentiva la investigación a estudiantes de pregrado con proyectos de iniciación científica (PIC). Igualmente, se establecen convocatorias para proyectos de investigación, de alto impacto, de incubación y de ciencia al servicio de la paz (Universidad Militar Nueva Granada, 2009).

En la Universidad de Antioquia, a través del Acuerdo Superior 204 del 6 de noviembre de 2001, se promulgó un principio de intercambio de investigadores con el fin de difundir los resultados de investigación. Esta universidad dispone de una Unidad de Transferencia de Tecnología, la cual ayuda a relacionar a los investigadores universitarios con el sector productivo (Universidad de Antioquia, 2011). Trasladar el conocimiento, mediante intercambio de investigadores, contribuye a obtener y apropiar experiencias de las universidades que están vinculadas.

La Universidad de Antioquia también cuenta con un Estatuto de Propiedad Intelectual. En ese estatuto, se estipulan lineamientos de normatividad de PI. Además, establece un Comité de Propiedad Intelectual para asesorar y divulgar, un manual de patentes como guía a los investigadores y una Revista UniversidadEmpresa-Estado en la que se motiva a realizar estas relaciones. La Universidad de Antioquia participa en convocatorias de infraestructura para la investigación, de 
Leonardo Andrés Briceño Marín, María Eugenia Morales Rubiano Desafíos de la política pública colombiana frente a la transferencia de resultados de investigación universitaria

movilidad para investigadores de Colciencias, de desarrollo experimental y demás (Universidad de Antioquia, 2011).

En ese mismo sentido, la Universidad del Valle participa en convocatorias externas de Colciencias y tiene convocatorias de investigación activas. Adicionalmente, cuenta con un banco de proyectos, estudios de género, en el área de humanidades, y beneficiarios de doctorado. En cuanto a normatividad, el Acuerdo 008 de 2006 del Consejo Superior define y reglamenta el Sistema de Investigaciones como plan de acción. El Acuerdo 023 de 2003 del Consejo Superior expidió el Estatuto sobre la Propiedad Intelectual. Por ende, la Universidad del Valle posee reglamentos sobre la Gestión de la Propiedad Intelectual, las convocatorias y la ejecución de proyectos de investigación. Para la gestión integral de calidad, tiene un proceso denominado investigación y generación de conocimiento, el cual se ilustra claramente con diagramas de flujo. También cuenta con una Oficina de Transferencia de Resultados de Investigación (OTRI) que se encarga de realizar actividades de vigilancia y comercialización tecnológica (Universidad del Valle, 2007).

\subsection{Universidades privadas}

Las relaciones de alianza permiten que una universidad con gran capacidad investigativa colabore con otra universidad de menor capacidad. De este modo, la primera universidad le transmite sus conocimientos a la segunda y la segunda recibe retroalimentación adecuada. Por ejemplo, la Corporación Universitaria Minuto de Dios se interesó por obtener alianzas estratégicas que propiciaran el aprendizaje y la transferencia de conocimiento. Por ello, se alió con la Universidad de los Andes. Entre estas dos universidades, se realiza intercambio de estudiantes (Cubides \& Vélez, 2010). Con esta alianza estratégica, la Corporación Universitaria Minuto de Dios espera convertirse en una universidad más competitiva.

El Centro Editorial UNIMINUTO se encarga de publicar todo tipo de producción intelectual desarrollada por la Corporación Universitaria Minuto de Dios. Además, esta universidad cuenta con convocatorias de investigación de desarrollo de investigación, semilleros de investigación, un sistema de investigaciones que regula 
la I+D+i y un Reglamento de Propiedad Intelectual (UNIMINUTO, 2014). En esta universidad se promueve el canal tradicional de transferencia.

Por otra parte, en la Pontificia Universidad Javeriana sede central, se creó la Vicerrectoría de Investigación en el año 2012. Aquello con el propósito de fortalecer la I+D. De la Vicerrectoría, se desprenden la Dirección de Investigación y la Dirección de Innovación. Esta última fomenta la cultura de trasferencia de tecnología, el apoyo para patentar y el emprendimiento universitario. Adicionalmente, realiza un trabajo conjunto con operadores de alianza universidad-empresa para fortalecer el desarrollo tecnológico en la región (Pontificia Universidad Javeriana, 2013). La reforma administrativa demuestra la importancia que tiene especializar las dependencias para la $\mathrm{I}+\mathrm{D}+\mathrm{i}$, el emprendimiento y la protección industrial.

En la Universidad Javeriana, se ha desarrollado spin off como resultado de investigación. También hay un área de consultoría para empresas públicas y privadas. En la Dirección de Innovación, se promueve el licenciamiento de patentes y la cultura del emprendimiento universitario (Pontificia Universidad Javeriana, 2013 citado en Briceño \& Morales, 2014).

Dentro de las políticas de esa universidad, se encuentra la Política de Investigación, la cual presenta principios y normas. La Estrategias para la Política de Investigación buscan un apoyo administrativo óptimo para la investigación. Existen convocatorias para innovación, movilidad, creación artística y para la movilidad (Pontificia Universidad Javeriana, 2014).

En la Universidad de los Andes, la financiación de la investigación es primordial. Por este motivo, esta universidad tiene convocatorias de programas de investigación, proyectos conjuntos en políticas públicas, artes e inter-facultades y convocatorias externas. Algunas de las convocatorias externas son de proyección internacional; por ejemplo, becas de postdoctorado en Japón. En el año 2007, la universidad creó la Vicerrectoría de Investigaciones. Además, cuenta con un reglamento de PI, en el que se conceptualiza términos, y hay regulación (Universidad de los Andes, 2012). 
Leonardo Andrés Briceño Marín, María Eugenia Morales Rubiano Desafíos de la política pública colombiana frente a la transferencia de resultados de investigación universitaria

La Universidad de la Sabana se trazó metas relacionadas con la investigación en su Visión 2019, la cual busca consolidar una cultura de investigación. Con el Acta 1233 del 12 de noviembre de 2009, se aprueba la política de PI de dicha universidad. En esa acta, se definen los conceptos y se decretan normas sobre la PI. En las políticas generales para la investigación en la Universidad de la Sabana, se busca lograr un posicionamiento con respecto al SNCTI del país. Ese objetivo planea alcanzarse mediante las políticas globales de investigación de la universidad. La Universidad de la Sabana tiene convocatorias internas de investigación científica y tecnológica. Además, participa en las convocatorias de Colciencias y de financiación a nivel mundial (Universidad de la Sabana, 2011).

\section{Dificultades QUe TIENEN las UNIVERSIDADES PÚBlicas Y PRIVADAS PARA TRANSFERIR}

En las universidades públicas, se presentan dificultades para crear un spin off. Éstas están relacionadas con: (a) el interés de investigadores y profesores en participar, (b) la retención del talento humano, (c) la sostenibilidad a lo largo del tiempo, (d) la administración de la financiación, y (e) el rechazo de las universidades a la idea de convertirse en empresas (Ruiz \& giraldo, 2012). De igual manera, los estímulos de inversión son bajos y no hay estructuras fuertes ni personal preparado para desarrollar una universidad emprendedora (Bonilla, 2012).

En las universidades privadas, la incentivación de los canales de transferencia depende de si éstos están definidos como objetivos institucionales y de que tengan un flujo de recursos dedicados a esos objetivos.

Por otra parte, las empresas pierden rentabilidad y no alcanzan la competitividad por la calidad de los estándares internacionales (Gómez \& Mitchell, 2014). Las empresas pueden no ver oportunidades de innovación en las universidades y se inclinan a importar la tecnología.

En Latinoamérica, la infraestructura física y social de las interacciones se encuentran en un estado inicial y no están fuertemente definidas (Pineda y Morales, 2010 citado 
en Pineda et al., 2011). Se deben doblar esfuerzos para que los canales de transferencia logren mejores resultados.

En el documento Colombia Visión 2019, se expresa que en Colombia, en el año 2006, la inversión en C y $\mathrm{T}$ era baja. Se admite que el gasto en actividades de I+i significa baja competitividad (Departamento Nacional de Planeación \& Colciencias, 2006). Aún, hoy en día, se observa escasa inversión, a pesar de los esfuerzos en pro de desarrollar estrategias para promover la $\mathrm{I}+\mathrm{D}+\mathrm{i}$.

El panorama actual no es alentador. La inversión en I+i es muy baja, en comparación al estándar internacional. La estructura administrativa de la universidad hace que el financiamiento sea muy complejo. En la universidad privada, no se refleja una rendición de cuentas clara, lo cual frena la innovación (OCDE, Banco Mundial \& Banco Internacional de Reconstrucción y Fomento, 2012). El sistema de innovación de Colombia es pequeño y débil porque el gasto en $\mathrm{I}+\mathrm{D}$ es del $0,2 \%$ del PIB. El registro de patentes también es bajo (OCDE, 2014). Se puede observar que las políticas públicas dirigidas a fortalecer la inversión en $\mathrm{I}+\mathrm{D}+\mathrm{I}$ no son eficientes. Por esta razón, faltan recursos para investigación en las universidades.

El ente financiador puede favorecer y/o obstaculizar la transferencia y apropiación por los recursos y el tiempo (Llanos \& Dager, 2003). En gran parte, los procesos de transferencia en las universidades deben depender de una entidad externa para financiar proyectos y lograr objetivos. Cuando los recursos para investigación son de fuentes propias y autosostenibles, es más eficiente.

Las instituciones receptoras de financiación de $\mathrm{C}$ y $\mathrm{T}$ y el Estado no tienen los niveles del alcance internacional; es necesario mejorar su capacidad (Corredor, 2012). Para que recursos brindados sean efectivos, se debe controlar en materia de los resultados de investigación. De modo similar, se deben buscar alternativas de obtención de recursos que impulsen a Colombia, comparándose con países desarrollados.

Ahora es difícil transferir el conocimiento porque hay obstáculos para articularlo con otros elementos o personas (Alfageme, Sáez, \& López, 2009). Los intereses y estructuras de los actores varían y al comunicarse se pueden presentar oposiciones. 
Leonardo Andrés Briceño Marín, María Eugenia Morales Rubiano Desafíos de la política pública colombiana frente a la transferencia de resultados de investigación universitaria

De hecho, existe una rigidez en la frontera político-administrativa entorno a la interacción de la universidad y el entorno (Pineda et al., 2011). La definición de una flexibilización administrativa en las instituciones y política por parte del gobierno facilita la participación de todos los actores.

Para transferir conocimiento hacia la sociedad efectivamente, la estructura organizacional y la cultura son fundamentales (Llanos \& Dager, 2003). Los factores externos influyen en la decisión para ser emprendedor. Por tal motivo, las organizaciones deben coordinar cómo lograr los objetivos (De Luco, 2014). La estructura actual de las instituciones públicas presenta dificultad para manejar los recursos, ya que la normatividad colombiana es compleja y demora los procesos. Por otro lado, la cultura debe relacionarse con un ambiente que promueva la investigación científica y los deseos de los actores por transferir conocimiento.

Cuando se genera un marco regulatorio incoherente de promoción tecnológica en las universidades, se dificulta la integración científica de Colombia al nivel internacional (Corredor, 2012). El país tiene la política del Sistema Nacional de Ciencia, Tecnología e Innovación, pero no ha consolidado una institucionalidad a largo plazo adecuada para la I+D, la C y la T. Por ahora, se observan grandes brechas entre Colombia y los países industrializados (Consejo Privado de Competitividad, 2013).

Colombia enfrenta aún grandes retos: (a) no ha avanzado hacia una economía basada en generación de conocimiento e innovación, (b) la inversión en $\mathrm{C}+\mathrm{T}+\mathrm{i}+\mathrm{I}+\mathrm{D}$ no ha aumentado en los últimos diez ańos, (c) el mayor porcentaje de las empresas colombianas manufactureras no son innovadoras y (d) el sector privado tiene poca inversión (Consejo Privado de Competitividad, 2013). Para empeorar la situación, las empresas no se preocupan por innovar, adquieren tecnologías importadas y adaptan los avances del extranjero.

En Latinoamérica, en general, la relación entre universidad-sector productivo es de alto nivel, pero baja con respecto a los países industrializados (Colciencias \& Consejo Nacional de Ciencia Y Tecnología (CNCyT), 2008). El éxito en la investigación de países extranjeros no se debe a la cantidad de estudiantes, sino a la transferencia de investigación. Lamentablemente, en Colombia, la colaboración entre centros 
de investigación del sector productivo y las universidades es bajo (Colombia, Departamento Nacional de Planeación \& Colciencias, 2006).

\section{CONCLUSIONES Y DISCUSIÓN}

En Colombia y en las universidades colombianas, las actividades de $\mathrm{C}+\mathrm{T}+\mathrm{i}$ tienen baja financiación y escaza capacidad de desarrollo. Adicionalmente, no se ve a la universidad como empresa. Por lo tanto, se debería diseñar una política pública específica que fortalezca la relación universidad-sector productivo y los canales de transferencia.

Actualmente, la administración pública interviene en la transferencia de conocimiento mediante financiación, diseño de leyes y estrategias. Las empresas que invierten en $\mathrm{I}+\mathrm{D}+\mathrm{i}$ y se relacionan con las universidades promueven la sostenibilidad en proyectos de C y T. Las políticas públicas que destinan los recursos para actividades de C y T impulsan la investigación, como parte fundamental para el desarrollo del país.

La reestructuración administrativa de las instituciones relacionadas con $\mathrm{C}$ y $\mathrm{T}$ en el país demuestra la importancia que tiene la investigación para tratar de generar desarrollo en la sociedad. En el futuro, el incremento en el desarrollo en $\mathrm{C}$ y $\mathrm{T}$ en Colombia se traducirá en disminución de la pobreza, mejora en la economía, aumento de productividad e incremento de las exportaciones sobre las importaciones.

Sin embargo, uno de los problemas más relevantes en la actualidad es la brecha entre la financiación de la transferencia universitaria a nivel internacional y la colombiana. En otros países, se observa que el éxito de la tecnología se debe al porcentaje de presupuesto de la nación destinado a actividades de C y T. Se debe dejar atrás el bajo interés por innovar en el sector productivo. El gobierno debe fortalecer mecanismos de alianza entre universidad-sector productivo para lógralo y se debe construir una cultura de $\mathrm{C}$ y $\mathrm{T}$ en la población. Con las relaciones universidadempresa-universidad, se asegura la capacidad del país para innovar, pero se debe dictaminar que los resultados de las políticas sean efectivos. Este dictamen se puede hacer mediante los indicadores de número de patentes desarrolladas y su progreso 
Leonardo Andrés Briceño Marín, María Eugenia Morales Rubiano Desafíos de la política pública colombiana frente a la transferencia de resultados de investigación universitaria

en la explotación comercial. Desde las universidades, se deben crear vínculos en el sector productivo. Para fortalecer la creación de spin off, se deben dar exenciones tributarias, ayuda económica y apoyo en consultoría administrativa.

El gobierno, las universidades, el sector productivo y la sociedad deben construir políticas públicas para responder al problema de la baja $\mathrm{I}+\mathrm{D}+\mathrm{i}$ en Colombia. Por una parte, debería comprobarse el avance en la ejecución. Para eso, no solo se deben formular objetivos transparentes sino que también debe establecerse si dichos objetivos son alcanzables y si realmente se han cumplido. De igual manera, se deberían fortalecer los programas de financiación para otorgar formación doctoral a los mejores estudiantes de las universidades y garantizarles buenas condiciones salariales y beneficios. De esta manera, se evitaría que los futuros investigadores e innovadores colombianos se fuguen a otros países.

Como se demostró en este artículo, al identificar algunas características de las políticas públicas de Colombia y de algunos países del mundo en cuanto a trasferencia de resultados de investigación universitaria, se contribuye a generar elementos para realizar contrastes frente a las teorías y formulaciones e implementaciones de éxito. Las políticas públicas de Colombia en materia de $\mathrm{I}+\mathrm{i}$ deben dirigirse a aumentar la inversión drásticamente en $\mathrm{C}$ y $\mathrm{T}$, a fomentar la colaboración de centros de investigación, y a crear redes de cooperación a través de Colciencias. Sin embargo, es necesario revisar las instituciones del gobierno para que el sector privado pueda estar representado.

Se concluye, por tanto, que en Colombia se buscan estrategias a través de las políticas de $\mathrm{C}$ y $\mathrm{T}$ similares a algunas de países desarrollados del mundo, pero los resultados no son los mismos. Lo anterior ocurre porque, hasta ahora, la orientación y el énfasis han estado en generar instrumentos de política para fomentar la innovación desde la oferta y la demanda. No obstante, faltan más instrumentos para la coordinación de estrategias y vinculaciones efectivas de los actores del Sistema Nacional de Ciencia, Tecnología e innovación. Igualmente, se requieren unas políticas que reconozcan tanto la diversidad de actores como las capacidades de cada uno. 
Por último, la normatividad de recursos y administración se debe flexibilizar para evitar obstáculos en la transferencia universitaria. Se debe institucionalizar la investigación colombiana con normatividad que facilite los procesos administrativos para generar competitividad en el país con alcance internacional.

\section{REFERENCIAS}

Aceytuno, M., \& Cáceres, F. (2009). Elementos para elaboración de un marco de análisis para el fenómeno de las spin-offs universitarias. Business And Economics--Economic Situation And Conditions, 23, 23-52.

Aceytuno, M.-T., \& Sánchez-López, C. (2014). Carrera académica y propensión a la transferencia de tecnología. El caso de la Universidad de Huelva. Investigaciones Regionales(28), 79-100.

Aguilar, L. (1993). La implementación de las Políticas. Mexico: Miguel Angel Porrua.

Alfageme, M. d., Sáez, P. L., \& López, J. E. (2009). La transferencia de conocimiento en la organización multiunidad: Un modelo integrado de análisis. Cuadernos de Estudios Empresariales(19), 43-73.

Bielschowsky, R. (2006). Evolución de las ideas de la CEPAL. Revista de la CEPAL, 50-62.

Bonilla, E. (2012). Hacia la constitución de spin off universitarias-Universidad Nacional de Colombia. ¿Pueden las universidades públicas crear Spin-Off? Encuentro de Vicerrectores de Investigación de Universidades Públicas. Medellin.

Briceńo, L., \& Morales, M. (2014). Características de la transferencia de conocimiento mediante patentes en una universidad privada colombiana. Encuentro Nacional de Investigación y Desarrollo ENID 2014 (págs. 1-6). Bogotá: Universidad Nacional de Colombia. 
Calderón-Martínez, M. G., \& García-Quevedo, J. (2013). Knowledge transfer and university patents in Mexico/Transferencia de conocimiento y patentes universitarias en México. Academia, 26(1), 33-60.

CESU. (21 de 10 de 2013). Política marco de investigación, ciencia, tecnología e innovación en el sector de la educación superior 2034. Bogotá: [documento de análisis preliminar. Versión no oficial].

Charry, G. P., \& Pérez, J. E. (2013). Las políticas públicas de desarrollo empresarial e innovación desde la perspectiva de la nueva gestión pública y la gobernanza: caso CREAME. Apuntes del CENES, 32(56), 175-204.

Chen-Chen, Y., Pei-Lee, T., Ooi, K.-B., \& Kim-Lan, S. (2009). Science and technology policy management in China. Journal of Technology Management in China, 4(1), 85-97.

Clark, B. (2001). The Entrepreneurial University: New Foundations for Collegia- lity, Autonomy, and Achievement. Journal of the Programme on Institutional Management in Higher Education, 13(2), 2-24.

Asamblea Nacional Constituyente. (1991). Constitución Política de Colombia.

Colciencias \& Consejo Nacional de Ciencia Y Tecnología (CNCyT). (agosto de 2008). Política nacional de fomento a la investigación y la innovación "Colombia construye y siembra futuro". [documento de trabajo].

Colciencias. (septiembre de 2010). Estrategia Nacional De Apropiación Social de la Ciencia, la Tecnología y la Innovación. Recuperado el 27 de octubre de 2014, de http://www. colciencias.gov.co/sites/default/files/ckeditor_files/files/ESTRATEGIA\% 20NACIONAL\%20DE\%20ASCTI_VFinal.pdf

Colciencias. (agosto de 2011). "Plan estrategico institucional 2011-2014". [documento de trabajo]. 
Colciencias. (21 de diciembre de 2012). Apropiación Social del Conocimiento. Recuperado el 27 de 10 de 2014, de http://www.colciencias.gov.co/programa_estrategia/ apropiaci-n-social-del-conocimiento

Colciencias. (octubre de 2013). Elementos para la elaboración de planes estratégicos de ciencia, tecnología e innovación. Bogotá: [documento de trabajo].

Congreso Nacional de la República. (26 de febrero de 1990). "Ley 29 del 27 de febrero de 1990, por medio de la cual se dictan disposiciones para el fomento de la investigación científica y el desarrollo tecnológico y se otorgan facultades extraordinarias”. En Diario Oficial, núm. 39205. 27 de febrero de 1990. Bogotá: Imprenta Nacional.

Congreso Nacional de la República. (22 de enero de 2009). "Ley 1286 del 23 de enero de 2009, por medio de la cual se modifica la Ley 29 de 1990, se transforma a Colciencias en Departamento Administrativo, se fortalece el Sistema Nacional de Ciencia, Tecnología e Innovación en Colombia y se dictan otras disposici. En Diario Oficial, núm. 47.241 de 23 de enero de 2009. Bogotá: Imprenta Nacional.

Congreso Nacional de la República. (16 de mayo de 2012). "Ley 1530 de 2012, por medio de la cual se regula la organización y el funcionamiento del Sistema General de Regalías. En Diario Oficial 48433 del 17 de mayo de 2012. Bogotá: Imprenta Nacional.

Consejo Privado de Competitividad. (21 de octubre de 2013). Informe nacional de competitividad 2013-2014. Bogotá.

Corbí, M. (1992). Proyectar la sociedad. Reconvertir la religión. Los nuevos ciudadanos. Barcelona: Editorial Herder.

Corredor, R. (2012). Gestión de los derechos de propiedad intelectual en el marco de modelos de colaboración científica: una oportunidad para las instituciones públicas de investigación en Colombia. Revista la Propiedad Inmaterial, 16, 5-17. 
Cubides, J. F., \& Vélez, O. L. (2010). Apropiación de conocimiento entre instituciones privadas de educación: transferencia del proyecto cup2 de uniandes a uniminuto. Journal of Information Systems and Technology Management: JISTEM, 33-70.

Dawson, R. (2000). Developing Knowledge-based Client Relationships: The Future of Profesional Services. Oxford: Ed. Butterwoth Heinemann.

De Luco, G. A. (2014). La intención emprendedora en estudiantes universitarios: el caso de la universidad de Deusto/entrepreneurial intention among university students: the case of the university of Deusto. Boletín de Estudios Económicos, 69(211), 151-172.

Departamento Nacional de Planeación \& Colciencias. (2006). 2019 Visión Colombia II Centenario: "fundamentar el crecimiento y el desarrollo social en la ciencia, la tecnología y la innovación, propuesta para discusión”. Imprenta Nacional de Colombia. [documento de trabajo].

Departamento Nacional de Planeación. (27 de 04 de 2009). Documento Conpes 3582: "Política Nacional de Ciencia, Tecnología e Innovación”. Bogotá: [documento de trabajo].

Departamento Nacional de Planeación. (octubre de 2011). "Estrategia Nacional de Innovación”. [documento de trabajo].

Departamento Nacional de Planeación. (2011). Plan Nacional de Desarrollo 2010-2014: "Prosperidad para todos". [documento de trabajo].

Departamento Nacional de Planeación, Colciencias \& ANSPE. (agosto de 2013). "Bases conceptuales de una política de innovación social". [documento de trabajo].

Drucker, P. (1993). Post-capitalist Society. Oxford: Betterworth-Hainemann.

Dutrénit, G., De Fuentes, C., \& Torres, A. (2010). Channels of interaction between public research organisations and industry and their benefits: evidence from Mexico. Science $\&$ Public Policy (SPP), 37(7), 513-526. doi:10.3152/030234210X512025 
Etzkowitz, H., \& Leydesdorff, L. (1995). The Triple Helix University Industry Gobernment Relations: A laboratory for Knowledge based Economic Development. EASST Review.

García, M. (2011). Políticas de innovación científica y tecnológica en América. Encrucijada, $1-12$.

Gómez, H., \& Mitchell, D. (marzo de 2014). Innovación y emprendimiento en Colombia: balance, perspectivas y recomendaciones de política, 2014-2018. Cuadernos de Fedesarrollo, número cincuenta.

Gross, C. M. (2013). The growth of China's technology transfer industry over the next decade: implications for global markets. Journal of Technology Transfer, 38(5), 716-747.

Herrera, D. M., \& Morales, J. L. (2014). El papel de las redes de actores en las políticas públicas de seguridad alimentaria y nutricional en la región del Urabá antioqueño, 2013. Estudios Politicos(45), 159-182.

Karnani, F. ( 2013). The university's unknown knowledge: tacit knowledge, technology transfer and university spin-offs findings from an empirical study based on the theory of knowledge. Journal of Technology Transfer, 38(3), 235-250.

Kindelán, M. P. (2013). Una perspectiva sobre el binomio enseñanza-investigación en la universidad del s. XXI/A perspective on teaching and investigation at the university of the 21st century. Revista Complutense de Educación, 24(1), 27-45.

Laredo, P. (2007). Toward a third mission for Universities. The third Mission of Universitiesworkshop. UNESCO.

Llanos, R. A., \& Dager, Y. B. (2003). Gestión de la integración social de los proyectos universidad-empresa un estudio de caso. Investigación \& Desarrollo, 11(2), 268-283.

Machikita, T., \& Ueki, Y. (2013). Knowledge transfer channels to Vietnam for process improvement. Management Decision, 51(5), 954-972. 
Martínez, I. R., \& Miranda, M. E. (2014). Las Spin-off Universitarias en Andalucía: Caracterización económico-financiera. Revista de Estudios Regionales, 75-101.

Melero, J. G., Angulo, P. S., \& Martín, J. J. (2011). La universidad ante el reto de la transferencia del conocimiento 2.0: análisis de las herramientas digitales a disposición del gestor de transferencia. Investigaciones Europeas de Direccion y Economia de la Empresa, 17(3), 111-126.

Ministerio de Economía, Fomento y Turismo. (2014). ¿Qué es la Transferencia de Tecnología o Transferencia Tecnológica? Recuperado el 03 de diciembre de 2014, de http://www. inapi.cl/portal/orientacion/602/w3-article-693.html

Ministerio de Educación Nacional. (20 de julio de 2009). Mineducacion. Recuperado el 31 de octubre de 2014, de http://www.mineducacion.gov.co/1621/w3-article-196467.html

Ministerio de Educación Nacional. (30 de septiembre de 2013). Lineamientos: "Política de educación Superior Inclusiva”. Bogotá: [documento de trabajo].

Muñoz, R. A. (2005). Las nuevas herramientas de la política de innovación: los sistemas de innovación y el desarrollo de clusters. Boletín de Estudios Económicos, 60(186), 413-429.

Muñoz, R. A. (2010). La competitividad sostenible: nuevo reto para las empresas y la sociedad1. Boletín de Estudios Económicos, 65(200), 263-281.

OCDE. (2014). Estudios de la OCDE de las Politicas de Innovación: Colombia. Resumen ejecutivo.

OCDE, Banco Mundial \& Banco Internacional de Reconstrucción y Fomento. (2012). Evaluación de Politicas Nacionales de Educación: La educación Superior en Colombia 2012.

OMPI. (2014). ¿Qué es una patente? Recuperado el 01 de 12 de 2014, de http://www.wipo. int/patents/es/ 
Parthasarathy, S. (2008). Science and Technology Policy in the United States: Open Systems in Action. Technology and Culture, 49(3), 819-820.

Perdomo, G. C., Arias, J. E., \& Lozada, N. B. (2013). Análisis de las políticas públicas de desarrollo empresarial e innovación desde la perspectiva de la nueva gestión pública y la gobernanza: caso Barcelona activa - incubadora de empresas. Perfil de Coyuntura Económica(21), 173-197.

Pestaña, M. J. (2013). Políticas de información: desarrollo y estrategias de los programas de la UE e impacto en Espańa. Revista General de Información y Documentación, 23(1), 9-25.

Pineda, K., Morales, M., \& Ortiz, M. (2011). Modelos y mecanismos de interacción universidad-empresa-Estado: reto para las universidades colombianas. Equidad y Desarrollo, 41-37.

Pontificia Universidad Javeriana. (junio de 2013). Informe del Rector al Consejo de Regentes 2012. Recuperado el 27 de julio de 2017, de http://issuu.com/javerianabogota/docs/ informe2012

Pontificia Universidad Javeriana. (2014). Investigación. Recuperado el 16 de diciembre de 2014, de http://www.javeriana.edu.co/investigacion/investigacion

Ramos, G. C. (2006). La competencia intercapitalista en ciencia y tecnología: quién es quien a principios del siglo XXI. Nómadas, 14(2), 3-27.

Rodríguez, F., Gómez, H., \& Valencia, J. (2013). Redes empresariales locales, investigación y desarrollo e innovación en la empresa. Cluster de herramientas de Caldas, Colombia. Estudios Gerenciales, 29(127), 247-257.

Roth, A.-N. (2002). Politicas públicas: formulación, implememntación y evaluación. Bogotá: Ediciones Aurora.

Rothwell, R. (1994). Towards the fifth-generation innovation process. International Marketing Review, 11(1), 7-31. 
Ruiz, O., \& giraldo, L. (2012). Fundamentación de las Spin Off. Basada en el estudio, reflexiones y experiencias de U de A en esta materia. ¿Pueden las universidades públicas crear Spin-Off? Encuentro de Vicerrectores de Investigación de Universidades Públicas. Medellín.

Sabato, J., \& Botana, N. (1968). La ciencia y la tecnología en el desarrollo futuro de América Latina. Revista de la Integración. Buenos Aires.

Sellenthin, M. O. (2009). Technology transfer offices and university patenting in Sweden and Germany. Journal of Technology Transfer, 34(6), 603-620.

UNCTAD. (24 de mayo de 2012). Examen de las Politicas de Ciencia, Tecnología e Innovación del Perú. Ginebra.

UNCTAD. (2012). Examen de las Políticas de Ciencia, Tecnología e Innovación: República Dominicana. Conferencia de las Naciones Unidas sobre comercio y desarrollo. Nueva York; Ginebra.

UNIMINUTO. (01 de diciembre de 2014). Investigación. Obtenido de http://www.uniminuto.edu/publicaciones

Universidad de Antioquia. (2011). Investigación. Recuperado el 02 de noviembre de 2014, de http://www.udea.edu.co/portal/page/portal/Programas/GruposInvestigacion/sistema UniversitarioInvestigacion/A.SUI

Universidad de la Sabana. (2011). Investigación. Recuperado el 15 de diciembre de 2014, de http://www.unisabana.edu.co/unidades/investigacion/pagina-principal/

Universidad de los Andes. (2012). Investigaciones. Recuperado el 14 de diciembre de 2014, de https://investigaciones.uniandes.edu.co/index.php/es/

Universidad del Valle. (2007). Vicerrectoria de Investigaciones Universidad del Valle. Recuperado el 15 de diciembre de 2014, de www.univalle.edu.co: http://darien.univalle.edu. $\mathrm{co} / /$ index.php?option=com_content $\&$ task=view\&id=15\&Itemid= 
Universidad Militar Nueva Granada. (2009). Investigación. Recuperado el 02 de novuembre de 2014, de http://www.umng.edu.co/web/guest/investigacion

Universidad Militar Nueva Granada. (2014). Acuerdos. Recuperado el 15 de 12 de 2014, de http://www.umng.edu.co/web/guest/acuerdos

Universidad Nacional de Colombia. (2005). Secretaria General, Estatuto General. Obtenido de http://www.unal.edu.co/estatutos/egeneral/anexo2.html

Universidad Nacional de Colombia. (2014). Investigación. Recuperado el 2 de 11 de 2014, de http://unal.edu.co/investigacion/la-investigacion-en-la-universidad/

Vizcarra, N. L., Torres, V. G., \& Ruiz, I. Y. (2014). Políticas públicas e incubadoras de empresas en México: estudio longitudinal 1994-2013. Global Conference on Business \& Finance Proceedings. 9, págs. 1115-1125. Hilo: Institute for Business \& Finance Research.

Xiwei, Z., \& Xiangdong, Y. (2007). Science and technology policy reform and its impact on China's. Technology in Society, 29, 317-325. 